\title{
Introduzindo o conceito de força do oscilador nas disciplinas iniciais de mecânica quântica
}

Introducing the concept of oscillator strength in the initial quantum mechanics courses

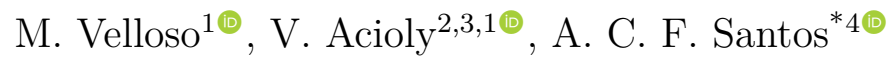 \\ ${ }^{1}$ Universidade Federal do Rio de Janeiro, PEMAT, PO 68530, CEP 21941-909, Rio de Janeiro, RJ, Brasil. \\ ${ }^{2}$ Escola Parque, CEP 22451-047, Rio de Janeiro, RJ, Brasil. \\ ${ }^{3}$ Colégio Santo Agostinho, CEP 22430-220, Rio de Janeiro, RJ, Brasil. \\ ${ }^{4}$ Universidade Federal do Rio de Janeiro, Instituto de Física, PO 68528, CEP 21941-972, Rio de Janeiro, RJ, Brasil.
}

Recebido em 21 de maio de 2020. Aceito em 21 de julho de 2020.

\begin{abstract}
Neste artigo, uma regra de soma para transições de elétrons entre os estados atômicos, proposta por Thomas, Reiche e Kuhn (TRK), apresentada apenas, e quando muito, em cursos avançados, é examinada de forma didática para o caso de transições entre estados de elétrons em dois sistemas familiares, os poços de potencial finito e infinito unidimensionais, tradicionalmente estudados em disciplinas introdutórias de mecânica quântica nos cursos de graduação em física.
\end{abstract}

Palavras-chave: força do oscilador, partícula em uma caixa, emissão de radiação.

In this paper, a sum rule for electron transitions between atomic states, proposed by Thomas, Reiche and Kuhn (TRK), presented only, and at most, in advanced courses, is examined in a didactic way for the case of transitions between states of electrons in two familiar systems, the one-dimensional finite and infinite potential wells, traditionally studied in introductory disciplines of quantum mechanics in undergraduate physics courses.

Keywords: oscillator strength, particle in a box, emission of radiation.

\section{Introdução}

Toda matéria, quer na fase gasosa, líquida ou sólida emite radiação, discreta ou contínua, cobrindo todo o espectro eletromagnético, embora em quantidades variáveis, eventualmente tão pequenas em algumas frequências, para alguns materiais e a algumas temperaturas que são indetectáveis com os instrumentos atuais. As estruturas de átomos e moléculas puderam somente ser reveladas através de fenômenos observáveis na escala macroscópica. Dentre estes fenômenos temos os espectros de emissão e absorção. Eles correspondem a eventos que ocorrem no interior dos átomos e moléculas e que nos ensinam sobre a sua estrutura. Assim, a espectroscopia é um estudo de importância fundamental em física e química.

Em 1885, Balmer encontrou uma fórmula empírica para linhas na faixa do visível [1]. Então, Lyman encontrou uma série correspondente no ultravioleta, e Paschen, Bracket e Pfund encontraram séries no infravermelho [1]. A falha da mecânica clássica na descrição destas séries levou Henri Poincaré a escrever: "Nosso primeiro olhar na distribuição das linhas nos faz pensar nos harmônicos que encontramos na acústica, mas a diferença é grande. As leis são simples, mas elas são de uma natureza intei-

*Endereço de correspondência: toni@if.ufrj.br ramente diferente.. e eu acredito que seja um dos mais importantes segredos da natureza" [2].

Nos cursos introdutórios de Mecânica Quântica, quando discutimos, por exemplo, o modelo atômico de Bohr, mencionamos que os átomos podem sofrer transições entre diferentes estados com as energias $\mathrm{E}_{\mathrm{a}}$ e $\mathrm{E}_{\mathrm{k}}$, quando um fóton com energia $\mathrm{h} \nu=\mathrm{E}_{\mathrm{k}}-\mathrm{E}_{\mathrm{a}}$ é absorvido ou emitido. Os experimentos mostram, no entanto, que o espectro de absorção ou emissão de um átomo não contém todas as frequências possíveis. Deve haver certas "regras de seleção" que selecionam entre todas as combinações possíveis $\mathrm{E}_{\mathrm{a}}$ e $\mathrm{E}_{\mathrm{k}}$ apenas aquelas entre as quais uma transição radiativa pode ocorrer. Além disso, a intensidade das linhas espectrais pode variar em muitas ordens de magnitude, o que significa que a probabilidade de uma transição geralmente depende fortemente da combinação específica dos dois estados atômicos.

Os dipolos oscilantes são as fontes mais importantes de radiação eletromagnética. Isso é verdade desde ondas de rádio com comprimentos de onda na faixa de metros ou quilômetros, que são associados a antenas macroscópicas até os raios X e gama, onde dipolos induzidos em átomos ou núcleos assumem o papel de antenas. Os dipolos podem ser induzidos por um campo externo deslocando a carga do centro de massa das distribuições de carga positiva e negativa, por exemplo, num átomo neutro. 
As probabilidades de transição para um problema ideal de um elétron, levam a alguns importantes teoremas gerais representados pelas chamadas regras de soma. Talvez a regra da soma mais conhecida na teoria atômica seja a regra da soma TRK [3-6]. Esta regra relaciona os termos dipolo entre os níveis energéticos $\mathrm{E}_{\mathrm{a}}$ e $\mathrm{E}_{\mathrm{k}}$, a saber

$$
\frac{2 m}{3 \hbar} \sum_{k} \omega_{k a}\left|r_{k a}\right|^{2}=1
$$

onde $\omega_{k a}=\frac{E_{k}-E_{a}}{\hbar}$ é a freqüência do fóton absorvido ou emitido, $\left|r_{k a}\right|^{2}=\left|\left\langle\psi_{k}|r| \psi_{a}\right\rangle\right|^{2}$ e $m$ sendo a massa do elétron.

Então, introduz-se uma quantidade adimensional $f_{k a}$, conhecida como a força do oscilador. Para um sistema de um único elétron, ela é definida como [3-5]:

$$
f_{k a}=\frac{2 m \omega_{k a}}{3 \hbar}\left|r_{k a}\right|^{2}
$$

No caso unidimensional, as equações 1 e 2 ficam, respectivamente, $\sum_{k} f_{k a}=\frac{1}{3} f_{\mathrm{e} a}=\frac{2 m \omega_{k a}}{3 \hbar}\left|x_{k a}\right|^{2}$.

Um ponto característico da regra de soma TRK original para os estados atômicos era sua falta de dependência do estado inicial das transições. As regras da soma têm desempenhado um papel importante no desenvolvimento de muitos ramos da física desde o primeiros dias da mecânica quântica [3-8].

Por exemplo, quando uma onda eletromagnética incidente do ar na superfície lisa do vidro, a onda incidente excita os elétrons das moléculas no vidro, que por sua vez irradiam ondas secundárias que se combinam para formar uma onda refletida resultante dada pela lei da reflexão e uma onda transmitida resultante dada pela lei de refração.

Santos [5] discutiu a origem clássica dos vários parâmetros que descrevem a intensidade das transições óticas em átomos e moléculas, com ênfase ao conceito de força de oscilador, que faz a ligação entre aqueles parâmetros.

Neste artigo, discutimos como a a regra de soma para TRK pode ser apresentada de forma didática para o caso de transições em dois sistemas familiares, os poços de potencial finito e infinito unidimensionais, tradicionalmente estudados em disciplinas introdutórias de mecânica quântica nos cursos de graduação em física. Na seção II discutimos o poço de potencial infinito e na seção III, o poço de potencial finito. Na seção IV apresentamos uma discussão dos resultados e como estes se relacionam com fenômenos físicos importantes. Finalmente, apresentamos nossas conclusões.

\subsection{Poço de Potencial Infinito}

O poço de potencial infinito (Fig. 1 a) é um modelo simples estudado nas disciplinas iniciais de mecânica quântica e muito utilizado, por exemplo, em química teórica para estimativas dos níveis de energia de moleculas que apresentam uma cadeia carbônica insaturada (pelo menos uma ligação dupla ou tripla, conhecidas como ligações pi) entre os átomos de carbono. Santos [9], usando
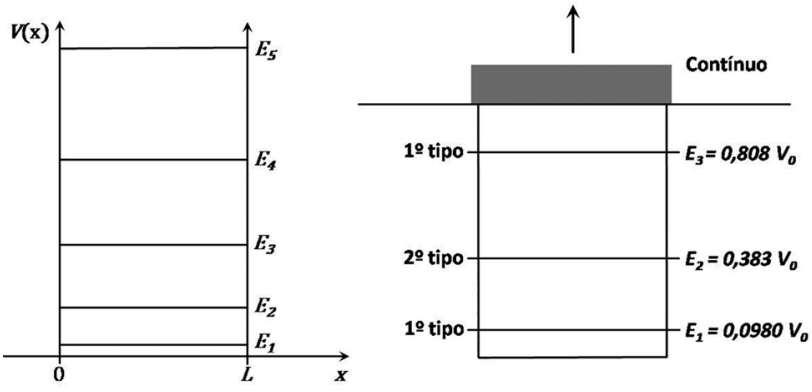

Figura 1: a) poço de potencial infinito e os seus respectivos cinco primeiros níveis de energia; b) poço de potencial finito de altura $\mathrm{V}_{\mathrm{O}}$.

o modelo de poço de potencial infinito, discutiu de forma didática a absorção da luz visível pelo pigmento visual rodopsina presente na retina [10], que contém o grupo retineno, um sistema conjugado, formado por átomos de carbono carbono simples e duplos, e funciona como uma antena de luz no processo de absorção de fótons. Por ser um modelo simples e bastante discutido em cursos de graduação em física e química, é um candidato perfeito para discutir a força do oscilador.

Assim, vamos discutir inicialmente o caso de um elétron estar confinado dentro de um intervalo finito $0 \leq \mathrm{x} \leq \mathrm{L}$ (Fig. 1a), num poço de potencial infinito. Isso pode ser realizado, se a energia potencial for escolhida como

$$
V(x)=\left\{\begin{array}{cc}
0 & 0 \leq x \leq L \\
\infty & x<0 \\
& x>L
\end{array}\right.
$$

Para calcular as possíveis energias da partícula com massa m, temos que resolver a equação de Schrödinger unidimensional independente do tempo com as condições de contorno apropriadas. Os níveis de energia $\mathrm{E}_{\mathrm{k}}$ e $\mathrm{E}_{\mathrm{a}}$ são dados por [1]:

$$
E_{k}=\frac{n_{k}^{2} \hbar^{2} \pi^{2}}{2 m L^{2}} E_{a}=\frac{n_{a}^{2} \hbar^{2} \pi^{2}}{2 m L^{2}}
$$

Os $\mathrm{n}_{\mathrm{k}}=1,2,3, .$. e $\mathrm{n}_{\mathrm{a}}=1,2,3, \ldots$ representam os números quânticos. Os auto-estados correspondentes são dados por [1]:

$$
\psi_{k}=\sqrt{\frac{2}{L}} \operatorname{sen}\left(\frac{n_{k} \pi x}{L}\right) \psi_{a}=\sqrt{\frac{2}{L}} \operatorname{sen}\left(\frac{n_{a} \pi x}{L}\right)
$$

Para calcularmos a força do oscilador, equação (2), vamos inicialmente calcular o valor esperado do operador posição x entre os dois auto-estados acima, que é dado por:

$$
x_{k a}=\frac{2}{L} \int_{0}^{L} \operatorname{sen}\left(\frac{n_{k} \pi x}{L}\right) x \operatorname{sen}\left(\frac{n_{a} \pi x}{L}\right) d x .
$$

Esta integral pode ser resolvida facilmente (vide o apêndice para uma tabela com as integrais envolvidas 
neste artigo). Pode-se mostrar que o resultado da integral acima será nulo a menos que

$$
n_{k}-n_{a}=\text { imparn }_{k}+n_{a}=\text { impar }
$$

Este é o primeiro resultado importante, raramente, para não dizer nunca discutido, nos cursos iniciais. As equações (7) representam uma regra de seleção para as transições entre os níveis de energia num poço de potencial infinito. Este resultado será mais discutido adiante. $\mathrm{O}$ resultado da integral na equação (6) para $\mathrm{x}_{\mathrm{ka}}$ é:

$$
x_{k a}=-\frac{8 L}{\pi^{2}} \frac{n_{k} n_{a}}{\left(n_{k}^{2}-n_{a}^{2}\right)^{2}}
$$

A força do oscilador (Eq. 2) fica:

$$
f_{k a}=\frac{64}{3 \pi^{2}} \frac{n_{k}^{2} n_{a}^{2}}{\left(n_{k}^{2}-n_{a}^{2}\right)^{3}}
$$

Note que $\mathrm{f}_{\mathrm{ka}}$ não está definido para $\mathrm{k}=\mathrm{a}$. Note também que $\mathrm{f}_{\mathrm{ka}}>0$ para $\mathrm{k}>\mathrm{a}$ (emissão) e $\mathrm{f}_{\mathrm{ka}}<0$ para $\mathrm{k}<\mathrm{a}$ (absorção). As figuras 2 e 3 mostram as forças do oscilador para as transições $\mathrm{n}_{\mathrm{a}}=1 \rightarrow \mathrm{n}_{\mathrm{k}}$ onde $\mathrm{n}_{\mathrm{k}}=2,4,6, \ldots$ e $\mathrm{n}_{\mathrm{a}}=$ $3 \rightarrow \mathrm{n}_{\mathrm{k}}$, onde $\mathrm{n}_{\mathrm{k}}=1,4,6, \ldots$ Como os $\mathrm{f}_{\mathrm{ka}}$ caem muito rápidamente com $\mathrm{n}$, é fácil verificar numericamente que $\sum_{k} f_{k a}=\frac{1}{3}$, usando por exemplo uma planilha EXCEL.

A relação entre probabilidades de transição e a descrição da mecânica quântica por elementos da matriz pode ser ilustrada de maneira simples por uma comparação com osciladores clássicos que emitem radiação eletromagnética. Um dipolo elétrico oscilante clássico com momento de transição de dipolo elétrico, no caso da partícula ser um elétron de carga de módulo e $=1,6 \times 10^{-19} \mathrm{C}$, é dado por

$$
\vec{D}=-e \vec{x}
$$

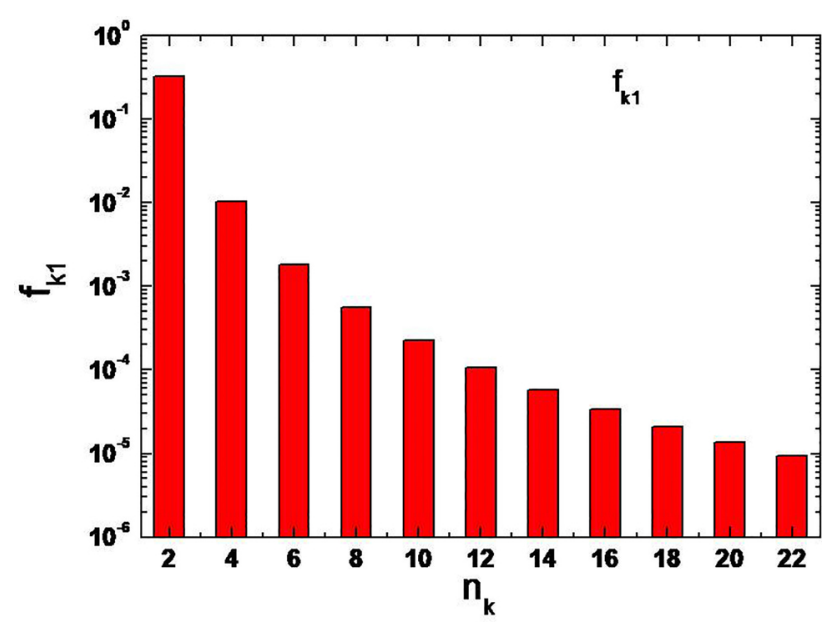

Figura 2: Força do oscilador $f_{k 1}$ para transição do estado fundamental $\mathrm{n}_{\mathrm{a}}=1$ para $\mathrm{n}_{\mathrm{k}}$ em função de $\mathrm{n}_{\mathrm{k}}$.

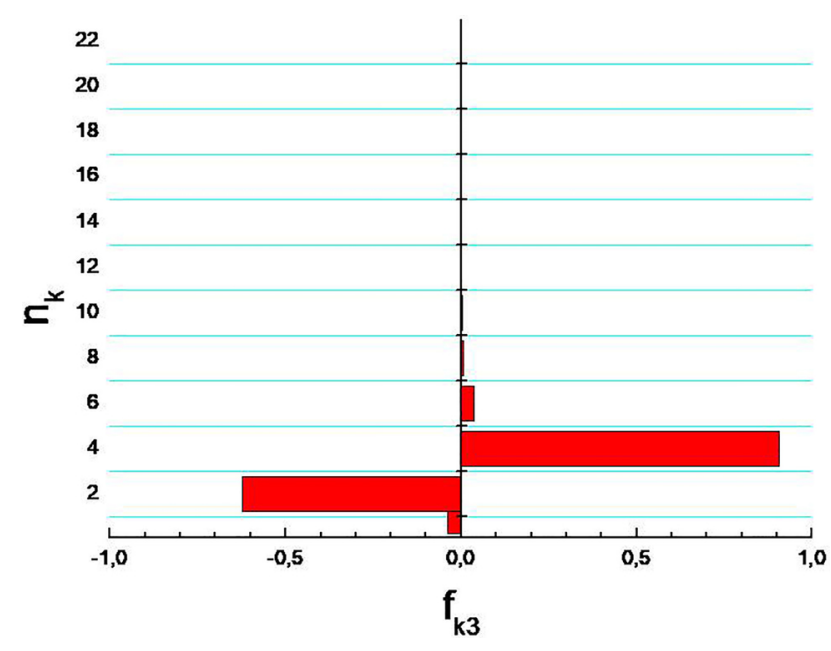

Figura 3: Força do oscilador $\mathrm{f}_{\mathrm{k} 3}$ para transição do estado $\mathrm{n}_{\mathrm{a}}=$ 3 para $\mathrm{n}_{\mathrm{k}}$. Os valores negativos de $\mathrm{fk}_{3}$ correspondem ao decaimento para um nível mais baixo, enquanto os valores positivos correspondem a transições para níveis superiores.

Ainda classicamente, a potência irradiada pelo dipolo é dada pela fórmula de Larmor $[5,11]$

$$
P_{\text {irradiada }}=\frac{\mu_{o} e^{2} \omega^{4}\langle\vec{x}\rangle^{2}}{6 \pi c}=\frac{\mu_{o} \omega^{4}\langle\vec{D}\rangle^{2}}{6 \pi c}
$$

Onde $\mu_{\mathrm{o}}$ é a permeabilidade magnética do vácuo e c a velocidade da luz, também no vácuo.

A potência de radiação emitida é, portanto, proporcional à média do momento dipolar ao quadrado. Durante a absorção ou emissão de um fóton, o átomo passa por uma transição entre dois níveis $\psi_{\mathrm{a}}$ e $\psi_{\mathrm{k}}$, alterando seu estado. A solução geral da equação de Schroedinger é [1]

$$
\psi(x, t)=\sum_{k} \psi_{k}(x) e^{-\frac{i E_{k} t}{\hbar}}
$$

O valor esperado do operador momento de dipolo elétrico é dado por:

$$
\begin{aligned}
& \langle\vec{D}\rangle=-e \int \psi^{*}(x, t) \vec{x} \psi(x, t) d x= \\
& -e \int \sum_{k} \psi *_{k}(x) e^{\frac{i E_{k} t}{\hbar}} \vec{x} \sum_{a} \psi_{a}(x) e^{-\frac{i E_{a} t}{\hbar}} d x
\end{aligned}
$$

Somente os termos com $\mathrm{k} \neq \mathrm{a}$ não se anulam na integral 13 . A razão é que $\psi^{*}(\mathrm{x}, \mathrm{t}) \psi(\mathrm{x}, \mathrm{t})$ é uma função par na coordenada $\mathrm{x}$, mas x é uma função ímpar. Como os limites da integral são de $\mathrm{x}=-\infty$ até $\mathrm{x}=+\infty$, a equação (13) se anula para integrandos ímpares. Assim, para uma transição $\mathrm{E}_{\mathrm{a}} \rightarrow \mathrm{E}_{\mathrm{k}}$, as funções de onda de ambos estados devem ser levadas em consideração, porque a probabilidade de transição depende de ambas as funções de onda. Portanto, definimos o valor esperado para o momento dipolo de transição $\mathrm{D}_{\mathrm{ka}}$ como a integral operador de momento de transição de dipolo elétrico entre os estados inicial $\psi_{\mathrm{a}}$ e final $\psi_{\mathrm{k}}$ :

$$
\vec{D}_{k a}=-e \vec{x}_{k a}
$$


onde vemos a relação do operador de momento de transição de dipolo com a força do oscilador ótico da equação (2). Podemos interpretar o momento de dipolo $\mathrm{D}_{\mathrm{ka}}$ como representando uma medida do solavanco que o elétron transmite ao campo eletromagnético quando realiza uma transição. Mais especificamente, grandes mudanças de carga, envolvendo grandes distâncias, transmite grandes impulsos eletromagnéticos desde que tenham um caráter dipolar, resultando em transições intensas. Além dessas transições de dipolo elétrico, também podem ocorrer transições de dipolo magnético muito mais fracas ou (ainda mais fracas) transições tipo quadrupolo elétrico. Classicamente, uma carga acelerada produz radiação. Isso pode ser melhor descrito por cargas que oscilam $\vec{r}(t)=\vec{r}_{o} e^{-i \omega t}$ e que dão origem a um dipolo oscilante $\vec{D}=-e \vec{r}=\vec{D}_{o} e^{-i \omega t}$, que por sua vez dá origem a uma densidade de corrente $\vec{j}=\rho \vec{v}$, onde $\rho$ é densidade volumar de carga e $\vec{v}=\frac{d \vec{r}}{d t}$ é a velocidade dos elétrons. O análogo quântico de $\rho$ é o produto -e $\psi^{*} \psi$ que representa a norma ao quadrado da função de onda multiplicada pela carga do elétron e a velocidade $\vec{v}=-i \omega \vec{r}$. A derivada temporal da densidade de corrente, sendo proporcional à aceleração da partícula, é a responsável pela emissão de radiação.

Através das equações (8) e (14), a expressão para o operador de momento de transição de dipolo elétrico para um elétron numa caixa é dada por:

$$
D_{k a}=-\frac{8 e L}{\pi^{2}}\left[\frac{n_{k} n_{a}}{\left(n_{k}^{2}-n_{a}^{2}\right)^{2}}\right]
$$

As equações 9 e 15 são obtidas a partir da chamada de aproximação dipolar [1,3] que é válida apenas quando o comprimento de onda $\lambda_{\mathrm{ka}}=\mathrm{hc} /\left(\mathrm{E}_{\mathrm{k}}-\mathrm{E}_{\mathrm{a}}\right)$ da radiação é grande em comparação com as dimensões do dipolo, $\mathrm{x}_{\mathrm{ka}}$. Para o caso de um elétron, isso é facilmente realizado desde que $\lambda_{\mathrm{ka}}$ seja muito grande em comparação com o tamanho médio $\mathrm{x}_{\mathrm{ka}}$ do dipolo emissor. Isso significa que

$$
\frac{\lambda_{k a}}{\left|x_{k a}\right|}>>\frac{m L c \pi^{2}}{h} \frac{\left(n_{k}^{2}-n_{a}^{2}\right)}{n_{k} n_{a}}
$$

que é facilmente realizado. Por exemplo, para a transição $\mathrm{n}_{\mathrm{a}}=1 \rightarrow \mathrm{n}_{\mathrm{k}}=2 \mathrm{e} \mathrm{L} \cong 10^{-10} \mathrm{~m}, \lambda_{\mathrm{ka}} / \mathrm{x}_{\mathrm{ka}} \cong 10^{2}$. As Eqs. 7 e 15 mostram que apenas transições eletrônicas durante as quais o momento de dipolo elétrico muda, contribuem para a absorção ou emissão de um fóton.

\section{O Poço de Potencial Finito}

Consideramos agora o caso do poço de potencial finito, ilustrado na parte b da Fig. 1. Este poço de potencial pode ser escrito matematicamente como:

$$
V(x)=\left\{\begin{array}{cc}
0 & -\frac{L}{2} \leq x \leq \frac{L}{2} \\
V_{o} \quad & x<-\frac{L}{2} \\
& x>\frac{L}{2}
\end{array}\right.
$$

Há dois tipos de soluções [1]. A primeira obedece à relação

$$
k_{I} \operatorname{tg}\left(k_{I} \frac{L}{2}\right)=k_{I I} k_{I}=\sqrt{\frac{2 m E}{\hbar}} k_{I I}=\sqrt{\frac{2 m\left(E-V_{o}\right)}{\hbar}}
$$

E a segunda, obedece à relação:

$$
k_{I} \cot g\left(k_{I} \frac{L}{2}\right)=-k_{I I}
$$

As soluções do primeiro tipo podem ser escritas como [1]:

$$
\psi(x)=\left\{\begin{array}{cc}
{\left[B \cos \left(\frac{k_{I} L}{2}\right) e^{\frac{k_{I I} L}{2}}\right] e^{k_{I I} x}} & x \leq-\frac{L}{2} \\
B \cos \left(\frac{k_{I} L}{2}\right) & -\frac{L}{2} \leq x \leq \frac{L}{2} \\
{\left[B \cos \left(\frac{k_{I} L}{2}\right) e^{\frac{k_{I I} L}{2}}\right] e^{-k_{I I} x}} & x \geq \frac{L}{2}
\end{array}\right.
$$

E as soluções do segundo tipo [1]:

$$
\psi(x)=\left\{\begin{array}{cc}
{\left[-A \operatorname{sen}\left(\frac{k_{I} L}{2}\right) e^{\frac{k_{I I} L}{2}}\right] e^{k_{I I} x}} & x \leq-\frac{L}{2} \\
A \operatorname{sen}\left(\frac{k_{I} L}{2}\right) & -\frac{L}{2} \leq x \leq \frac{L}{2} \\
{\left[A \operatorname{sen}\left(\frac{k_{I} L}{2}\right) e^{\frac{k_{I I} L}{2}}\right] e^{-k_{I I} x}} & x \geq \frac{L}{2}
\end{array}\right.
$$

De modo a simplificar a discussão, adotamos os valores que constam no apêndice G da ref. [1], ou seja, consideramos o caso onde $\sqrt{\frac{m V_{o} L^{2}}{2 \hbar^{2}}}=4$, que apresenta três níveis de energia:

$$
\begin{array}{ccc}
E_{1}=0,0980 V_{o} & \text { tipo } & 1 \\
E_{2}=0,383 V_{o} & \text { tipo } & 2 \\
E_{3}=0,808 V_{o} & \text { tipo } & 1
\end{array}
$$

Os auto-estados correspondentes aos três níveis de energia são, respectivamente [1]:

$$
\psi_{1}(x)=\left\{\begin{array}{cc}
17,9 \frac{1}{\sqrt{L}} e^{3,80 \frac{x}{a_{/ 2}}} & x \leq-\frac{L}{2} \\
1,26 \frac{1}{\sqrt{L}} \cos \left(1,25 \frac{x}{L_{/ 2}}\right) & -\frac{L}{2} \leq x \leq \frac{L}{2} \\
17,9 \frac{1}{\sqrt{L}} e^{-3,80 \frac{x}{a_{/ 2}}} & x \geq \frac{L}{2}
\end{array}\right.
$$$$
\psi_{2}(x)=\left\{\begin{array}{cc}
-18,6 \frac{1}{\sqrt{L}} e^{3,16 \frac{x}{L_{/ 2}}} & x \leq-\frac{L}{2} \\
1,23 \frac{1}{\sqrt{L}} \operatorname{sen}\left(2,47 \frac{x}{L_{/ 2}}\right) & -\frac{L}{2} \leq x \leq \frac{L}{2} \\
18,6 \frac{1}{\sqrt{L}} e^{-3,16 \frac{x}{L / 2}} & x \geq \frac{L}{2}
\end{array}\right.
$$

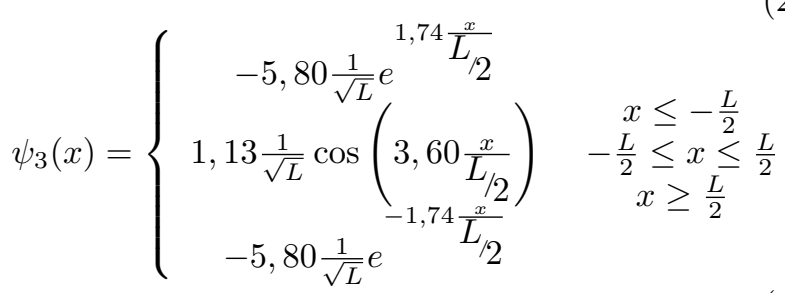


Repetimos agora os cálculos análogos aos da seção anterior, desta vez para o poço de potencial finito. Para facilitar, apresentamos no apêndice as integrais necessárias. As figuras 4, 5 e 6 apresentam os respectivos integrandos para os valores esperados $x_{\mathrm{ka}}$ em função da coordenada $\mathrm{x}$ para as transições entre os três níveis de energia, a saber, $1 \rightarrow 2,1 \rightarrow 3$ e $2 \rightarrow 3$. Note que os integrandos para as transições $1 \rightarrow 2$ e $2 \rightarrow 3$ representam funções pares na coordenada $\mathrm{x}$, enquanto o integrando para a transição $1 \rightarrow 3$ representa uma função ímpar. É fácil perceber que as integrais $\mathrm{x}_{12}$ e $\mathrm{x}_{23}$ são não nulas, enquanto a integral $\mathrm{x}_{13}$ é nula. Obtemos assim, as regras de transição para os níveis de energia do poço de potencial finito. Note que os resultados para o poço de potencial finito e infinito devem coincidir para o limite $\mathrm{V}_{\mathrm{o}} \rightarrow \infty$. A Tabela 1 apresenta os valores para as forças do oscilador $f_{\text {ka }}$ para as transições do poço de potencial finito. É fácil perceber também que as $\mathrm{f}_{\mathrm{ka}}$ obedecem à regra de soma TKR, com exceção de $\mathrm{f}_{\mathrm{k} 3}$. Podemos entender esta violação devido ao fato do número finito de estados para este sistema. No caso do terceiro nível, não há níveis discretos acima de $\mathrm{E}_{3}$, então , se o elétron se encontra no terceiro nível, ele apenas pode decair, a menos que seja ionizado para o contínuo.

$$
\begin{aligned}
& \sum_{k} f_{k 1}=f_{21}+f_{31}=\frac{1}{3} \\
& \sum_{k} f_{k 2}=f_{32}+f_{12}=\frac{1}{3}
\end{aligned} \quad \sum_{k} f_{k 3}=f_{13}+f_{23}=-\frac{2}{3}
$$

\section{Discussão}

Como vimos nas seções anteriores, a lei da conservação da energia é um requisito necessário, mas não suficiente para tornar uma transição possível. A razão para isso é que, além de conservação de energia, a conservação do momento angular e certas regras de simetria também devem ser obedecidas, dependendo do sistema. Tudo isso

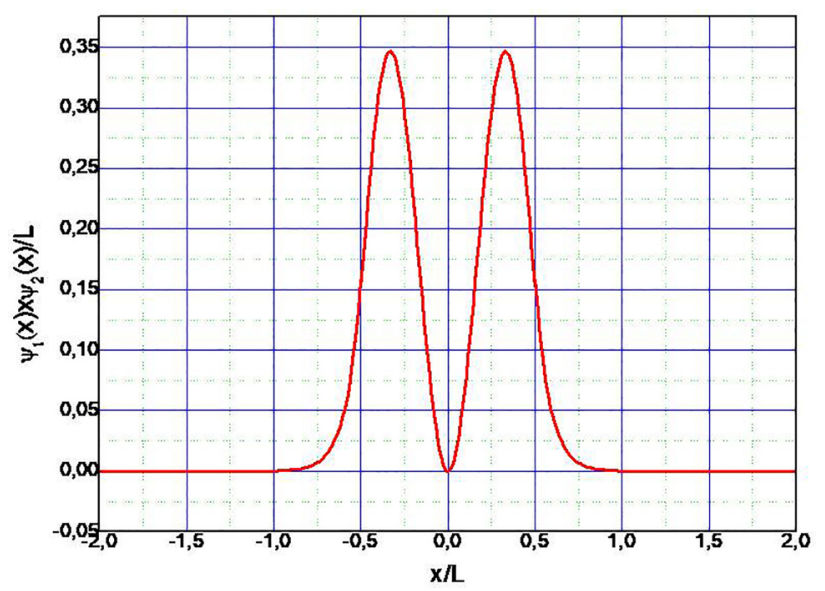

Figura 4: Gráfico da função $\psi_{1}(\mathrm{x}) \times \psi_{2}(\mathrm{x}) / \mathrm{L}$ em função da coordenada $x / L$. Como a função é par, resulta numa integral não nula.

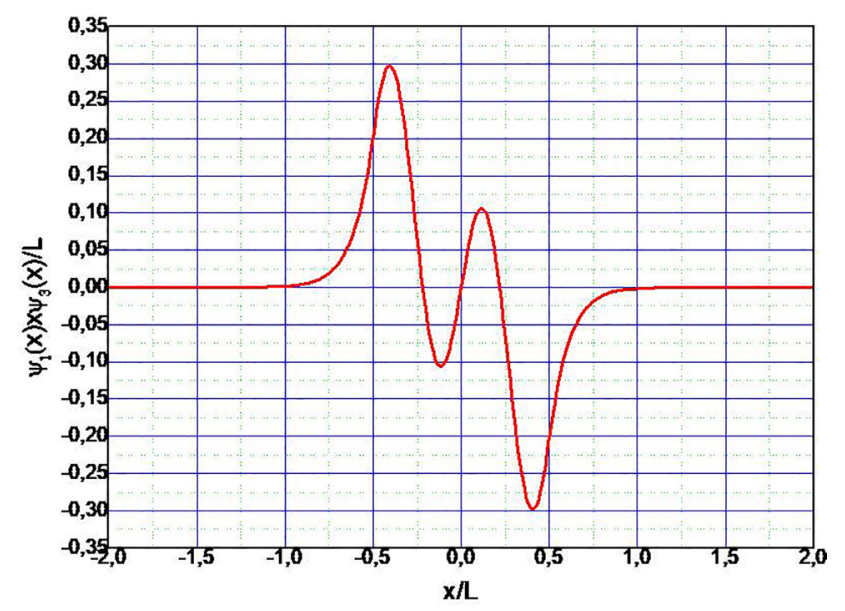

Figura 5: Gráfico da função $\psi_{1}(\mathrm{x}) \times \psi_{3}(\mathrm{x}) / \mathrm{L}$ em função da coordenada $x / L$. Como a função é ímpar, resulta numa integral nula.

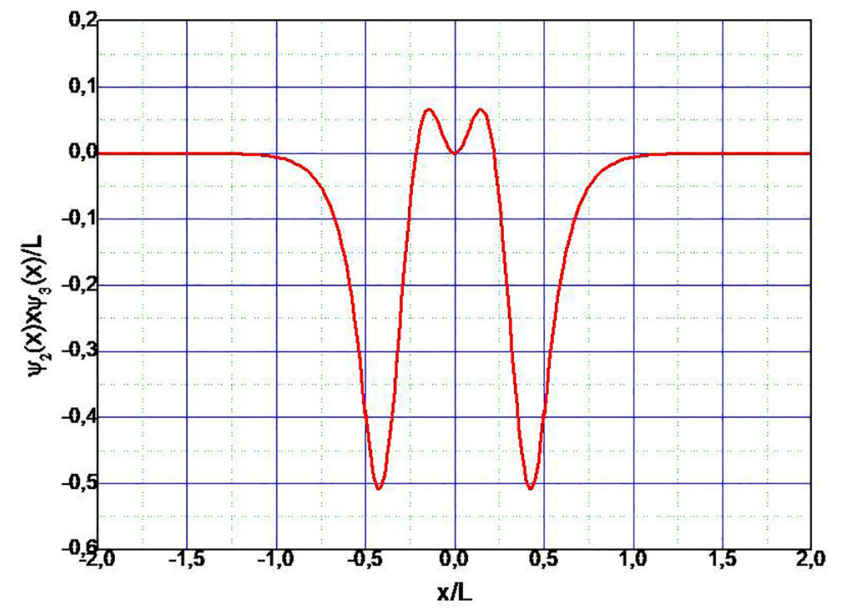

Figura 6: Gráfico da função $\psi_{2}(\mathrm{x}) \times \psi_{3}(\mathrm{x}) / \mathrm{L}$ em função da coordenada $x / L$. Como a função é par, resulta numa integral não nula.

Tabela 1: Valores para as forças de oscilador para transições entre os estados discretos do poço de potencial finito.

\begin{tabular}{llccc}
\hline $\begin{array}{l}\text { Estado inicial } \\
\left(\psi_{\mathbf{a}}\right)\end{array}$ & $\begin{array}{l}\text { Estado } \\
\left(\psi_{\mathbf{k}}\right)\end{array}$ & Final & processo & $\mathbf{f}_{\text {ka }}$ \\
\hline$\psi_{1}$ & $\psi_{2}$ & absorção & 0,33 \\
\hline$\psi_{1}$ & $\psi_{3}$ & absorção & 0 \\
\hline$\psi_{2}$ & $\psi_{3}$ & absorção & 0,66 \\
\hline$\psi_{2}$ & $\psi_{1}$ & emissão & $-0,33$ \\
\hline$\psi_{3}$ & $\psi_{1}$ & emissão & 0 \\
\hline$\psi_{3}$ & $\psi_{2}$ & emissão & $-0,66$ \\
\hline
\end{tabular}

está incluído nos elementos de matriz de transição dipolar (eq. 14) .

Durante uma transição do estado estacionário inicial $\psi_{\mathrm{a}}$ para o estado estacionário de final $\psi_{\mathrm{k}}$, também conhecido como quantum jump, a posição média do elétron oscila entre os dois estados a uma frequência igual à diferença de energias $\omega_{\mathrm{ka}}=\left(\mathrm{E}_{\mathrm{k}}-\mathrm{E}_{\mathrm{a}}\right) / \hbar$, para $\mathrm{E}_{\mathrm{K}}>\mathrm{E}_{\mathrm{a}}$ ou $\omega_{\mathrm{ka}}=$ $\left(\mathrm{E}_{\mathrm{a}}-\mathrm{E}_{\mathrm{k}}\right) / \hbar$, para $\mathrm{E}_{\mathrm{a}}>\mathrm{E}_{\mathrm{k}}$. Durante a transição, o elétron 
está em um estado misto, no qual a probabilidade de encontrá-lo no estado inicial diminui gradualmente da unidade para zero, enquanto a probabilidade de encontrálo no estado final aumenta durante o período de transição do mesmo. Durante a transição, o elétron normalmente executa milhões de oscilações.

No caso das transições de um elétron em uma camada externa do átomo, a diferença de energia em $\mathrm{E}_{\mathrm{k}}-\mathrm{E}_{\mathrm{a}}$ equivale a alguns elétron-volts. A frequência de transição cai então na região espectral entre o infravermelho próximo e o ultravioleta próximo $(1,0 \mathrm{eV}$ corresponde a um comprimento de onda $\lambda$ de $1,234 \mathrm{~m}=$ infravermelho próximo, enquanto $3,0 \mathrm{eV}$ corresponde a $\lambda=478 \mathrm{~nm}$ $=$ região espectral do azul). Essas transições originam principalmente a emissão de luz visível e são chamadas de transições óticas. Por outro lado, se um elétron em uma camada atômica interna de um átomo multielerônico é excitado em estados desocupados, sua energia de excitação pode ser da ordem de vários keV. Quando retorna ao seu estado inicial, a radiação com comprimentos de onda curtos (raios-X) são emitidos. O espectro de emissão ou absorção correspondente à essas transições, portanto, caem entre a região ultravioleta distante e a região dos raios $\mathrm{X}$ com comprimentos de onda entre 0,01 a $50 \mathrm{~nm}$ (comprimento de onda de $0,01 \mathrm{~nm}$ corresponde a uma energia de cerca de $120 \mathrm{keV}$ ).

No caso das moléculas, estas podem ter momentos de dipolo elétrico, induzidos pelo campo elétrico incidente ou permanente como conseqüência de sua estrutura. Embora as moléculas sejam eletricamente neutras, seus centros de carga positiva e negativa podem não coincidir.

As transições de um estado inicial para um estado final ocorrem quando ambas as funções de onda dão origem a uma amplitude de oscilação não nula $\mathrm{x}_{\mathrm{ka}}$, conforme indicado pelo elemento da matriz na Eq. (6). Se as funções de onda inicial e final forem tais que a integral na Eq. (6) é não nula, a transição será permitida. Examinando a integral, verificamos que a coordenada x é ímpar (substituir $\mathrm{x}$ por $-\mathrm{x}$ altera o sinal do integrando), exigindo que as funções de onda inicial e final sejam de paridade oposta (uma par, outra ímpar nas coordenadas de integração) para resultar uma integral diferente de zero.

Por exemplo, a vibração simétrica de alongamento na molécula $\mathrm{CO}_{2}$ (linear com o átomo de carbono ocupando a posição central) é dita inativa no infravermelho, porque o momento dipolar , Eq. 14, permanece zero durante toda essa vibração. No entanto, também existem moléculas simétricas (como $\mathrm{H}_{2} \mathrm{O}$ que possui estrutura na forma de "V" e por isso um momento de dipolo permanente) nas quais todas as vibrações normais são ativadas por infravermelho porque o momento dipolo muda durante todas elas. Na linguagem da teoria de grupos, dizemos que a integral 14 corrresponde a uma representação totalmente simétrica de um grupo de ponto.

\section{Conclusões}

Em suma, neste artigo, apresentamos como a regra de soma TKR para transições de elétrons entre os estados atômicos, proposta por Thomas, Reiche e Kuhn, pode ser aplicada de forma didática para o caso de transições entre estados de elétrons em dois sistemas familiares, tradicionalmente estudados em disciplinas introdutórias de mecânica quântica nos cursos de graduação em física. Esperamos assim contribuir para a contextualização do estudo destes sistemas e ao mesmo tempo discutir temas que são apresentados somente em cursos avançados, mas são importantes para a compreensão do mundo em que vivemos.

\section{Material suplementar}

O seguinte material suplementar está disponível online: Apêndice: Integrais utilizadas no texto

\section{Referências}

[1] R. Eisberg e R. Resnick, Física Quântica, Átomos, Moléculas, Sólidos, Núcleos e Partículas (Campus, Rio de Janeiro, 1988), $4^{\mathrm{a}}$ ed.

[2] L. de Broglie, The Revolution in Physics, a nonmathematical survey of quanta (The Noonday Press, New York, 1958).

[3] B.H. Bransden e C.J. Joachain, Physics of atoms and molecules (Person Education, Essex, 2002), $2^{\mathrm{a}}$ ed.

[4] G.C. Schatz e M.A. Ratner, Quantum Mechanics in Chemistry (Dover, Mineola, New York, 2001).

[5] A.C.F. Santos, Revista Brasileira de Ensino de Física 42, e20190176 (2020).

[6] R.C. Hilborn, American Journal of Physics 50, 982 (1982).

[7] M. Belloni e R.W. Robinett, American Journal of Physics 76, 798 (2008).

[8] E. Hadjimichael, W. Currie e S. Fallieros, American Journal of Physics 65, 335 (1997).

[9] A.C.F. Santos, Revista Brasileira de Ensino de Física 32, 2303 (2010).

[10] R.W. Schoenlein, L.A. Peteanu, R.A. Mathies e C.V. Shank, Science 254, 412 (1991).

[11] D.J. Griffiths, Eletrodinâmica (Pearson, São Paulo, 2011), $3^{\mathrm{a}}$ ed. 